\title{
DESIGN AND PERFORMANCE OF A BALANCED SINGLE-ENDED DUAL-FED DISTRIBUTED POWER AMPLIFIER
}

\author{
Kimberley W. Eccleston \\ Department of Electrical and Computer Engineering, \\ National University of Singapore, Singapore 119260 \\ E-mail: elekwe@nus.edu.sg
}

\begin{abstract}
In this paper we demonstrate a design method for the balanced amplifier employing two singleended dual-fed distributed power amplifiers. The method ensures that all FET output power is utilised, all FETs have equal output power at the centre frequency and operate into identical, optimum loadlines. The amplifier has improved efficiency compared to the conventional distributed amplifier and has improved input and output match compared to the dual-fed distributed amplifier.
\end{abstract}

\section{Introduction}

The single-fed (conventional) distributed amplifier has had limited success in power applications due to low efficiency stemming from fundamental causes such as uneven output power distribution among the FETs, and output power wastage in the back-ward waves. A variation to the distributed amplifier (DA) topology, the dual-fed DA [1] (shown in Fig. 1(a)), has been previously proposed as a means to utilise output power in both the backward and forward waves [1-4]. The previous work [1-4] essentially only considered a small-signal analysis and small electrical spacing between FETs. D'Agostino \& Paolino [2][3] did show simulation results for output power and efficiency but did not provide large-signal design guidelines.

Recent work by this author [5][6] considered large electrical spacing between the FETs, and determined the conditions whereby power equalisation among the FETs can be achieved at microwave frequencies. With power equalisation it is possible to have all FETs operating into an optimum loadline [6]. Eccleston [5] also demonstrated that dual-feeding offers significant compensation for gate and drain line attenuation that is known to limit the performance of the single-fed DA.

Unfortunately the dual-fed DA has inherently high input and output port mismatch [7], despite the results reported by D'Agostino \& Paolino [2][3]. Moazzam \& Aitchison [7] have shown that the port match can be improved using a balanced configuration employing two quadrature hybrids and two so-called single-ended dual-fed DAs. However, Moazzam \& Aitchison [7] only considered an amplifier with 2 FETs and did not consider large-signal design.

It is therefore the purpose of this work to develop design guidelines for the single-ended dual-fed distributed power amplifier that employs an even number of FETs. The design guidelines ensure that the all FETs operate into their optimum loadline, and that all output power is utilised, thereby maximising efficiency. This paper will also demonstrate the application of the guidelines to the design of a balanced amplifier employing two single-ended dual-fed distributed power amplifiers.

\section{Dual-Fed Distributed Power Amplifier}

The circuit diagram of the dual-fed DA is shown in Fig. 1(a) for the case of four FETs. The input power is split equally by the input hybrid and fed to both ends of the gate line, and the output hybrid combines waves appearing at both ends of the drain line. Fig. 1(b) shows its equivalent circuit, with the input hybrid replaced by equivalent input voltage sources presented to the gate line and the output hybrid replaced by loads presented to the drain line. Normally the input voltage sources have identical amplitude but have phase difference, $\phi$. 

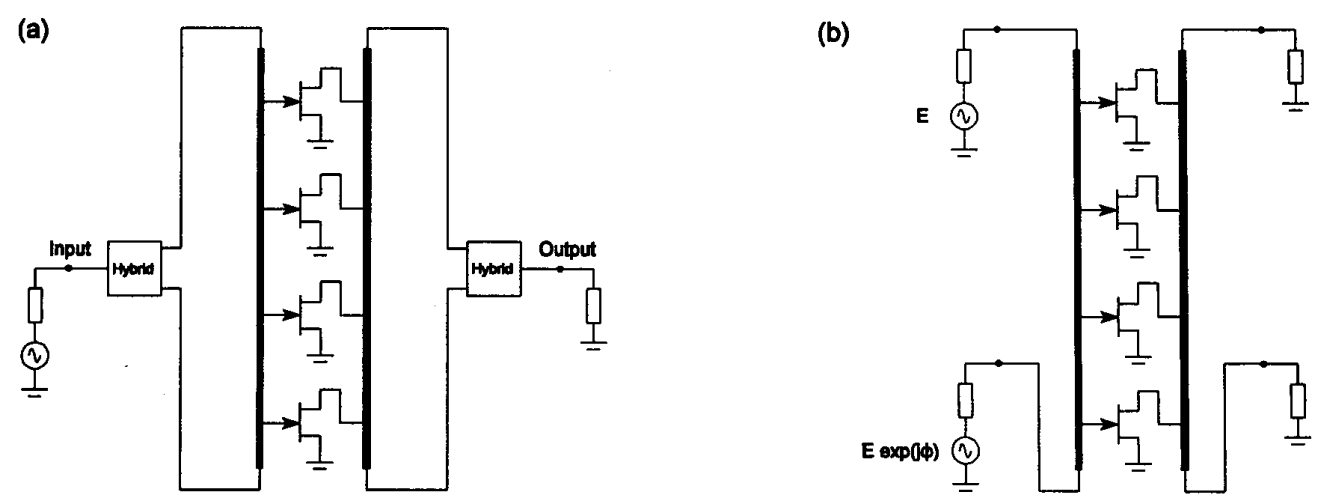

Fig. 1 Dual-fed distributed amplifier (a) and its equivalent circuit (b).

If the FETs are electrically spaced by $180^{\circ}$ (at the centre frequency), all FETs have identical output power for anti-phase input voltages $\left(\phi\right.$ equal to $\left.180^{\circ}\right)[5]$. nb. anti-phase input voltages also offers the best gain advantage over the conventional single-fed DA [4]. Eccleston [6] has shown for the case of an even number $(n)$ of FETs spaced $180^{\circ}$ and anti-phase feeding ( $\phi$ equal to $180^{\circ}$ ), the optimum characteristic impedance of the drain line is given by:

$$
Z_{o_{\text {opt }}}=2\left(\frac{V_{D_{\max }}-V_{D_{\min }}}{I_{D_{\max }} n}\right)
$$

where $V_{D \max }$ and $V_{D \min }$ are the maximum and minimum drain voltages, and $I_{D \max }$ is the maximum drain current so that the FET is operated to the fullest and safest extent within the saturation region under class A conditions. The bandwidth of such operation is limited to about $30 \%$ [5]. Parallel resonators at both the input and output of each FET can be used to suppress harmonics as well as absorb FET input and output capacitances [6].

\section{Single-Ended Dual-Fed Power Distributed Amplifier}

The poor input and output match of the dual-fed DA arises since two ports of both hybrids are essentially connected together by a transmission line rather than terminated with isolated loads. Interestingly, losses on the gate and drain lines results in improvement of the input and output match, and would explain the good input and output match reported by D'Agostino \& Paolino [2][3].

With reference to Fig. 1(b), for the case of an even number of FETs spaced $180^{\circ}$ and antiphase input signals ( $\phi$ equal to $180^{\circ}$ ), it can be shown that the voltage midpoint along both the gate and drain lines are zero. Hence these midpoints can be shorted to earth, as shown in Fig. 2, without disturbing the operating conditions of the FETs. It is apparent that the dual-fed distributed amplifier is now divided into two separate distributed amplifiers (so called single-ended dual-fed DAs [7]) and hence both ends of the gate and drain lines are isolated. $\mathrm{nb}$. the short-circuit gate and drain line terminations must be located $90^{\circ}$ from the nearest FET.

It can be shown that the FETs in the two single-ended dual-fed DAs operate under essentially the same conditions as though they were in a dual-fed DA with an even number of FETs spaced $180^{\circ}$ and with antiphase input feeding. [With the amplifier divided into two halves, the phase difference between the input signals need not be $\phi$ as in Fig. 1(b).] Hence (1) is still applicable for calculating the optimum characteristic 
impedance of the drain line with $n$ being the total number of FETs contained in both single-ended dual-fed DAs. Quadrature hybrids can then be used to combine these two single-ended dual-fed DAs in a balanced configuration [7] as shown in Fig. 3 and hence achieve good input and output port match. The short-circuit terminations for the gate and drain lines (which are $90^{\circ}$ from the nearest FET) also provide convenient dc bias application.

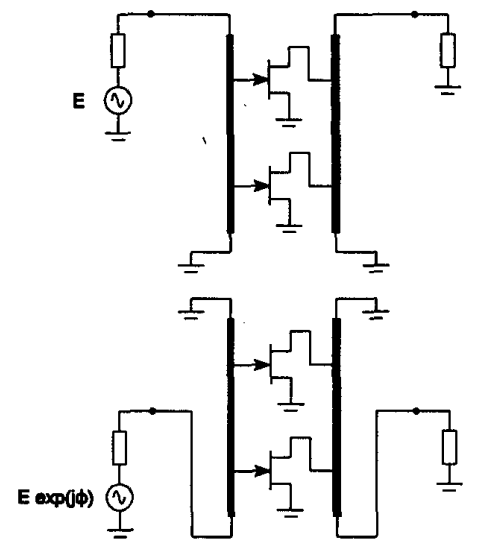

Fig. 2 Pair of single-ended dual-fed distributed amplifiers with four FETs spaced $180^{\circ}$.

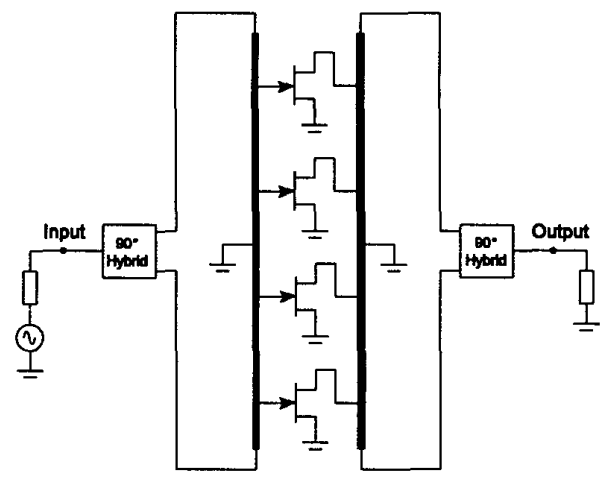

Fig. 3 Balanced amplifier employing a pair of single-ended dual-fed distributed amplifiers with four FETs spaced $180^{\circ}$.

\section{Design Example}

We consider the design of a single-ended dual-fed DA with a centre frequency of $10 \mathrm{GHz}$ employing four Fujitsu FLK012XP FETs, and branch-line $\left(90^{\circ}\right)$ hybrids at both the input and output. The FETs have a minimum and maximum drain voltage of $1 \mathrm{~V}$ and $10 \mathrm{~V}$ respectively, and a maximum drain current of $60 \mathrm{~mA}$. The FETs were each spaced $180^{\circ}$ at $10 \mathrm{GHz}$ so as to achieve power equalisation at $10 \mathrm{GHz}$ [5]. Using (1) the optimum value of the output line characteristic impedance is $75 \Omega$ which was coupled to the output hybrid by quarter-wave transformers. The de gate and drain bias voltages were set to $-1 \mathrm{~V}$ and $5.5 \mathrm{~V}$ respectively for class A operation. The input line characteristic impedance was set to $50 \Omega$. The input and output of each FET was resonated using parallel resonators with $Q$ chosen to trade-off distortion and bandwidth. The gate and drain bias voltages were fed to the midpoints of the gate and drain lines, therefore providing the shortcircuits indicated in Fig. 2 and 3. Further calculations revealed that the theoretical output power of each FET to be $68 \mathrm{~mW}(18.3 \mathrm{dBm})$, and hence a total output power of $272 \mathrm{~mW}(24.3 \mathrm{dBm})$, and drain efficiency of 41 $\%$.

Simulations using HP MDS ${ }^{\mathrm{TM}}$ were then used to check the validity of the calculations and their corresponding assumptions. The FETs were modelled using a Statz-Raytheon based model. At $10 \mathrm{GHz}$ with an input level of $12 \mathrm{dBm}$, each FET sourced $17.6 \mathrm{dBm}$, and the total amplifier output power was $23.5 \mathrm{dBm}$ meaning all FET output power was utilised. The drain efficiency was found to be $34 \%$, whilst the power added efficiency was $31 \%$. Fig. 4 shows the small-signal frequency responses of the gain, and the input and output reflection coefficients. It is clear that the ports show good match over the amplifier passband. Fig. 5 shows the output voltage waveform at $10 \mathrm{GHz}$ for an input power of $12 \mathrm{dBm}$. The load trajectories of the each FET/resonator pair were found to be similar and nearly optimum over a frequency range $9.2 \mathrm{GHz}$ to 10.8 $\mathrm{GHz}$ and are identical at $10 \mathrm{GHz}$. The simulation therefore confirmed the design procedure. The distortion of the loadlines (from straight lines) observed is mainly due to the presence of even harmonics in the FET/resonator currents that are shunted to ground via the short circuits rather than the capacitances in the resonators. 


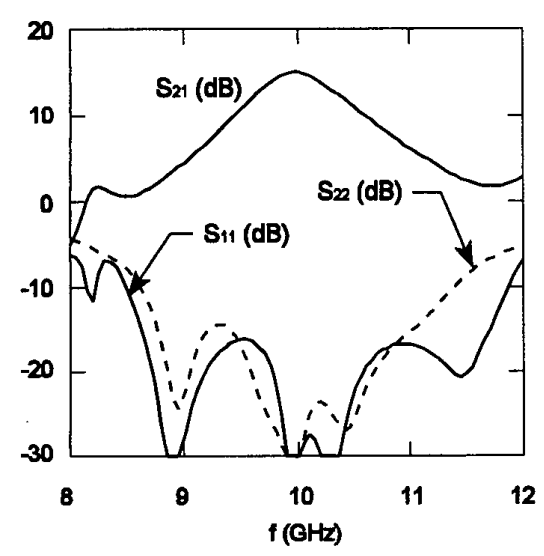

Fig. 4 Small-signal frequency responses of gain and port reflection coefficients.

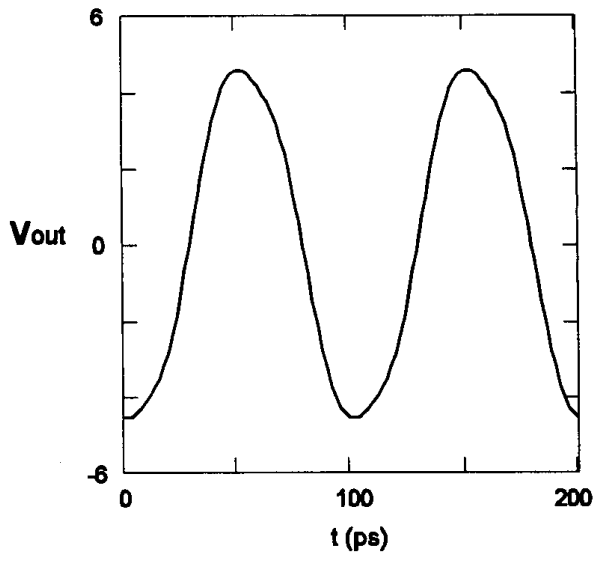

Fig. 5 Output voltage waveform when the input power is $12 \mathrm{dBm}$.

\section{Conclusion}

In this paper we have shown the development of a design method for a balanced amplifier employing a pair of single-ended dual-fed distributed power amplifiers that achieves power equalisation among their FETs, full utilisation of FET output power, and optimum loadline for all FETs. For power equalisation at the centre frequency, the FETs must be spaced $180^{\circ}$ and the short-circuit terminations of the gate and drain lines must be $90^{\circ}$ from the nearest FET. And for an optimum loadline, the characteristic impedance of the drain lines must be $2 / n$ times the optimum load line resistance of one of the FETs, where $n$ (even) is the total number of FETs. Simulations have demonstrated the validity of the design method. The configuration not only has improved efficiency compared to the conventional single-fed distributed amplifier but has improved input and output match compared to the dual-fed distributed amplifier.

\section{References}

[1] C. S. Aitchison, et al, "The Dual-Fed Distributed Amplifier", 1988 IEEE Int. Microwave Symp., MTT-S, pp $911-914$

[2] S. D'Augustino \& C. Paoloni, "Power Distributed Amplifier Based on Interdigital Combiners", Electronics Letters, 29(23), 11 Nov 1993, pp 2050 - 2051.

[3] S. D'Augustino \& C. Paoloni, "Design of a High-Performance Power-Distributed Amplifier Using LangeCouplers", IEEE T. on MTT, 42(12), Dec 1994, pp 2525 - 2530.

[4] A. Botterill \& C. S. Aitchison, "Effect of hybrids on gain performance of dual-fed distributed amplfiers", Electronics Letters, 30(13), 23 June 1994, pp 1067 - 1068.

[5] K. W. Eccleston, "Output Power Performance of Dual-Fed and Single-Fed Distributed Amplifiers", Microwave and Optical Tech. Letters, 27(4), Dec 20, 2000, pp $281-284$.

[6] K. W. Eccleston, Design Considerations for the Dual-Fed Distributed Power Amplifier", 2000 Asia-Pacific Microwave Conference, 3 - 6 Dec 2000, Sydney, Australia, pp 205 - 208.

[7] M. R. Moazzam \& C. S. Aitchison, "A High Gain Dual-Fed Single Stage Distributed Amplifier", 1994 IEEE Int. Microwave Symp., MTT-S, pp 1409- 1412. 